\title{
"Chronic sensory demyelinating neuropathy": chronic inflammatory demyelinating polyneuropathy presenting as a pure sensory neuropathy
}

\author{
Shin J Oh, Juan L Joy, Reha Kuruoglu
}

\begin{abstract}
The clinical electrophysiological and histological features of 10 cases of "chronic sensory demyelinating neuropathy" (CSDN) are reported. This entity is characterised by: 1) subacute or chronic progression; 2) pure sensory neuropathy; 3) high spinal fluid protein in the majority of cases; 4) electrophysiological evidence of demyelination affecting motor as well as sensory nerve fibres; 5 ) demyelination on sural nerve biopsy and 6) good response to immunotherapy in progressive phase. It is believed that this entity represents chronic inflammatory demyelinating polyneuropathy (CIDP) presenting as pure sensory neuropathy.
\end{abstract}

$(\mathcal{F}$ Neurol Neurosurg Psychiatry 1992;55:677-680)

In 1988 we reported "sensory neuropathy as a variant of chronic inflammatory demyelinating neuropathy" in the abstract form. ${ }^{1}$ We report here the details of the clinical, electrophysiological and histological features of this entity, which we termed "chronic sensory demyelinating neuropathy (CSDN)".

\section{Patients and methods}

Ten patients were examined over a period of 18 years. One case (case 8) was previously reported. $^{2}$ All had clinically pure sensory peripheral neuropathy and normal muscle strength upon careful examination. Chronic inflammatory demyelinating polyneuropathy (CIDP) patients with mixed sensory-motor neuropathy but with clear sensory predominance were excluded. Patients with acute sensory neuropathy or with subacute sensory neuropathy due to dorsal root ganglion lesions were excluded. Patients with known causes of neuropathy were also excluded. These include diabetes, ureamia, alcoholism, vitamin deficiency, heavy metal intoxication, collagen vascular diseases, thyroid disease, malignancy, and hereditary neuropathy. One patient (case 7) with transient benign gammopathy is included. Patients were followed for periods ranging from one to seven years.

Routine nerve conduction studies were performed and abnormalities were recognised following previously described methods and criteria. ${ }^{3}$ Conduction block was considered to be present when the peak-to-peak amplitude was reduced by more than $40 \%$ compared to the distal value (fig 1). Abnormal temporal dispersion was considered to be present when the CMAP shape was abnormal with multiple phases (more than four) and total duration longer than three standard deviations above the normal mean value (fig 1 ). ${ }^{3}$ In five patients, the plantar nerves were studied using the nearnerve needle sensory nerve conduction technique. ${ }^{4}$ Needle EMG examination was carried out in all cases.

Sural nerve biopsy was performed in eight cases. Frozen sections were stained with modified trichrome, cresyl-fast violet, hematoxylin and eosin, and crystal violet stains. Paraffin sections were stained with hematoxylin and eosin and congo-red stains. Semithin EM sections in three cases were stained with toluidine blue. Teased nerve preparations were studied in four cases.

To compare the degree of sensory impair-

A.

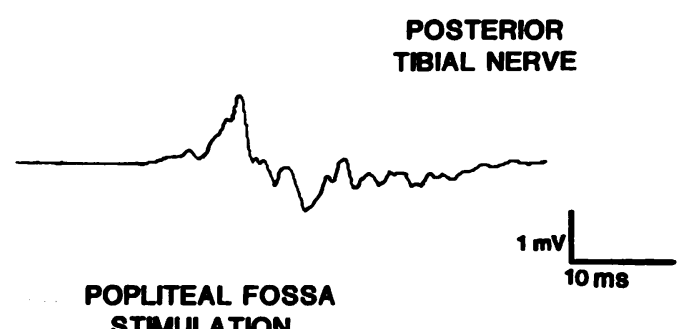

B.

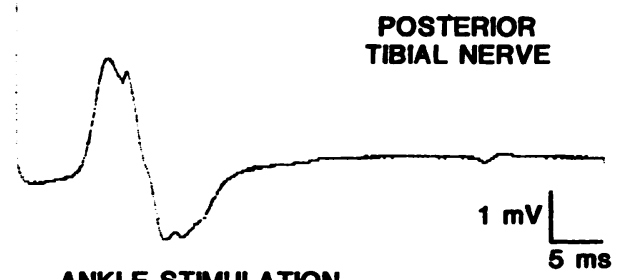

ANKLE STIMULATION

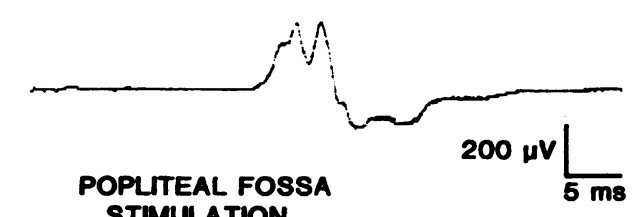

Figure Motor nerve conduction. A) Abnormal temporal dispersion in a CMAP in posterior tibial nerve with stimulation at the popliteal fossa in case 8. B) Conduction block in posterior tibial nerve in case 1.

Department of
Neurology, The
University of Alabama
at Birmingham, The
Veterans
Administration
Medical Center,
Birmingham,
Alabama, USA
S J Oh
J L Joy
R Kuruoglu
Correspondence to:
Dr Oh, Department of
Neurology, The University of
Alabama at Birmingham,
UAB Station, Birmingham,
Alabama 35294 , USA
Received 5 August 1991
and in revised form
6 November 1991.
Accepted 13 November
1991


Table 1 Clinical feature

\begin{tabular}{|c|c|c|c|c|c|c|c|}
\hline $\begin{array}{l}\text { Case } \\
\text { number }\end{array}$ & Sex/age & $\begin{array}{l}\text { Duration of } \\
\text { progression }\end{array}$ & Sensory deficit & Reflexes & $\begin{array}{l}\text { CSF protein } \\
\text { (mg/dl) }\end{array}$ & Treatment & Final status \\
\hline $\begin{array}{r}1 \\
2 \\
3 \\
4 \\
5 \\
6 \\
7 \\
8 \\
9 \\
10\end{array}$ & $\begin{array}{l}M / 48 \\
M / 28 \\
M / 62 \\
M / 49 \\
M / 53 \\
M / 55 \\
M / 59 \\
M / 62 \\
M / 53 \\
M / 65\end{array}$ & $\begin{array}{l}6 \text { months } \\
6 \text { years } \\
10 \text { years } \\
1 \text { years } \\
1 \text { year } \\
1.5 \text { months } \\
1 \text { year } \\
4 \text { months } \\
8 \text { months } \\
2 \text { years }\end{array}$ & $\begin{array}{l}\text { SP, 31 } \\
\text { Painful SP, } 4 \\
\text { Painful SP, } 6 \\
\text { SP, } 3 \\
\text { SP, 5 } \\
\text { MNM, 2 } \\
\text { Painful SP, } 4 \\
\text { SP, } 7 \\
\text { SP, } 2 \\
\text { Ataxic SP, } 7\end{array}$ & $\begin{array}{l}++1-^{2} \\
++ \\
- \\
++ \\
++ \\
++1- \\
++ \\
++ \\
++1-\end{array}$ & $\begin{array}{l}74, \text { OCB } \\
37 \\
55, \text { OCB } \\
63 \\
39, \text { OCB }^{\star} \\
139 \\
108\end{array}$ & $\begin{array}{l}\text { P, improved } \\
\text { Propoxyphene } \\
\text { Am/narcotic } \\
\text { P, improved } \\
\text { P, improved } \\
\text { Am/narcotic } \\
\text { P, A, \& PE, improved } \\
\text { P, A, \& PE, improved }\end{array}$ & $\begin{array}{l}\text { Stable } \\
\text { Stable } \\
\text { Stable } \\
\text { Stable } \\
\text { Steroid dependent } \\
\text { Normal } \\
\text { Stable } \\
\text { Normal with steroid } \\
\text { Stable } \\
\text { Stable with steroid }\end{array}$ \\
\hline
\end{tabular}

'Neurological disability score: 0 , normal; 10 , maximal disability. ${ }^{2}++$, normal; + , decreased; - , absent. Abbreviations: $M$, male; SP, symmetrical polyneuropathy; MNM, mononeuropathy multiplex; P, prednisone; A, azathioprine; Am, amitriptyline; PE, plasma exchange; OCB, oligoclonal band.

^Transient benign monoclonal gammapathy was present.

ment, we used the peripheral nerve disability scoring system which we reported previously. ${ }^{5}$ According to this system, 0 is normal and 10 is maximally disabled in the sensory functions. The disability score is the sum of the scores of two different modalities: pin-prick and vibration/position sense.

\section{Results}

Clinical features There were ten male patients, ranging in age from 28 to 65 years (table 1). All these patients had a slowly progressive monophasic course. Duration of progression from onset to maximal disability ranged from four months to 10 years. None had had any antecedent illness, or vaccination.

The most common initial symptom, observed in eight cases, was numbness in the feet. Numbness in the hands was the initial symptom in two cases and numbness in the entire arm in two cases. In three cases, pain was also described in addition to numbness. Gradually, these sensory symptoms spread to the distal parts of the limbs, culminating in a symmetrical stocking-glove distribution in most cases. In one case (6), there were complaints of numbness in the left T8-10 dermatomes. In one case (10), unsteadiness was the chief complaint at the time of first evaluation because of sensory ataxia.

Although the degree of impairment varied, nine cases showed the classic finding of sensory polyneuropathy: sensory impairment in "stocking-glove distribution," worse distally

Table 2 Motor Nerve Conduction

\begin{tabular}{|c|c|c|c|c|}
\hline Nerves & Median & Ulnar & Peroneal & Post tibial \\
\hline $\begin{array}{l}\text { Normal } \\
\text { No potential } \\
\text { Low CMAP } \\
\text { Prolonged terminal latency }^{2} \\
\quad>150 \% \text { of normal means }{ }^{\star \star}\end{array}$ & $\begin{array}{l}1^{\star} \\
0 \\
1 \\
4 \\
4\end{array}$ & $\begin{array}{l}0 \\
0 \\
1 \\
5 \\
2\end{array}$ & $\begin{array}{l}0 \\
0 \\
7 \\
8 \\
6\end{array}$ & $\begin{array}{l}0 \\
0 \\
6 \\
8 \\
5\end{array}$ \\
\hline $\begin{array}{l}\text { Slow nerve conduction velocity: } \\
\text { Distal segment } \\
\text { Proximal segment }^{4} \\
<60 \% \text { of normal means } \\
\text { A } \\
\text { Abnormal temporal dispersion } \\
\text { Conduction block } \\
\text { Prolonged F-wave } \\
>150 \% \text { of normal means } \\
\quad{ }^{\star \star}\end{array}$ & $\begin{array}{l}5 \\
3 \\
2 \\
1 \\
1 \\
8 \\
3\end{array}$ & $\begin{array}{l}5 \\
5 \\
2 \\
0 \\
1 \\
8 \\
3\end{array}$ & $\begin{array}{r}10 \\
9 \\
7 \\
7 \\
5 \\
10 \\
7\end{array}$ & $\begin{array}{r}10 \\
2 \\
8 \\
2 \\
10 \\
4\end{array}$ \\
\hline
\end{tabular}

${ }^{\star}$ Number of cases. ${ }^{\star \star}$ These were considered to indicate demyelination.

Abbreviations: CMAP, compound muscle action potential. TL, terminal latency. NCV, nerve conduction velocity. 'Normal lower limits for the CMAP amplitude: $5 \mathrm{mV}$ for median, ulnar, and posterior tibial nerves; 4 for peroneal nerve. ${ }^{2}$ Normal upper limits for the terminal latency: 3.6 msec for median; 2.5 for ulnar; 4.8 for peroneal; $5 \cdot 1$ for posterior tibial nerves. ${ }^{3}$ Normal lower limits for the NCV: $48.2 \mathrm{~m} / \mathrm{sec}$ for median; 42.8 for ulnar; 41.9 for peroneal; 40.6 for posterior tibial nerves. ${ }^{4}$ Normal lower limits for the NCV: $56.0 \mathrm{~m} / \mathrm{sec}$ for median; 52.7 for ulnar; $39 \cdot 1$ for peroneal nerves. ' Normal upper limits for the F-wave latency: $29.7 \mathrm{msec}$ for median; 30.3 for ulnar; 55.5 for peroneal; 57.3 for posterior tibial nerves. and symmetrically. In one case (6), sensory multiple mononeuropathy was an appropriate label because of sensory impairment in fingers, toes, and the left T8-10 dermatome. In 9 cases, pinprick and proprioception sensations were affected equally. In one case, proprioception loss was severe enough to produce sensory ataxia. In one case (8), almost the entire body was hypoalgesic, including the trigeminal area. Three patients could be classified as having painful sensory neuropathy because of severe pain. Muscle stretch reflexes were impaired in six cases: diffuse areflexia in three and distal areflexia or hyporeflexia in three. In four cases, reflexes were normal. Muscle strength was normal in all cases. Essential tremor (case 5) and fasciculations (case 9) were each noted in one case respectively.

Spinal fluid findings Protein was elevated in five patients, ranging from $55-139 \mathrm{mg} / \mathrm{dl}$, and normal in two. Lymphocytes and glucose were normal in all tested cases. In three cases, oligoclonal bands were positive. In one of these three, IgG paraprotein was also present in the serum once.

Serum auto-antibodies In four tested cases, autoantibodies for $M A G, G_{1}$, asialo- $G_{1}$ and $\mathrm{Gal}(\beta 1-3)$ GalNAc were normal.

Nerve conduction findings In all cases, there was evidence of diffuse peripheral neuropathy. Nerve conduction abnormalities were present in all cases in lower extremities and in eight cases in upper extremities. Motor nerve conductions were abnormal in all cases and just as prominent as sensory nerve conduction abnormalities though all our cases had a clinically pure sensory neuropathy. The most common abnormalities were a prolonged $\mathrm{F}$-wave latency and slow motor nerve conduction velocity (NCV) (table 2).

Electrophysiological evidence of demyelination (abnormal temporal dispersion, conduction block, more than $150 \%$ prolongation of normal means in terminal and $\mathrm{F}$-wave latencies, and/or NCV slower than $60 \%$ of normal means) was not widespread but was present in two or more nerves in all cases, confirming demyelinating neuropathy (fig 1). Conduction block was observed in six cases, abnormal temporal dispersion in nine, marked slowing of NCV (NCV less than $60 \%$ of normal mean) in seven, and more than $150 \%$ prolongation of normal means in terminal and F-wave latencies in six cases each. Sensory nerve conduction was abnormal in all cases (table 3 ). The 
Table 3 Sensory and Mixed Nerve Conduction

\begin{tabular}{|c|c|c|c|c|}
\hline & No potential & $\begin{array}{l}\text { Low } \\
\text { amplitude' }\end{array}$ & $\begin{array}{l}\text { Slow } \\
N C V^{2}\end{array}$ & Normal \\
\hline $\begin{array}{l}\text { Sensory Nerve Conduction: } \\
\text { Median } \\
\text { Ulnar } \\
\text { Sural } \\
\text { Medial plantar } \\
\text { Lateral plantar }\end{array}$ & $\begin{array}{l}0 \star \\
0 \\
3 \\
3 \\
3\end{array}$ & $\begin{array}{l}2 \\
3 \\
0 \\
2 \\
0\end{array}$ & $\begin{array}{l}5 \\
4 \\
5 \\
1 \\
0\end{array}$ & $\begin{array}{l}4 \\
4 \\
2 \\
0(N=5) \\
1(N=4)\end{array}$ \\
\hline $\begin{array}{l}\text { Mixed Nerve Conduction: } \\
\text { Median W-E } \\
\text { E-A } \\
\text { Ulnar W-E } \\
\text { E-A }\end{array}$ & $\begin{array}{l}0 \\
0 \\
0 \\
0\end{array}$ & $\begin{array}{l}3 \\
0 \\
2 \\
0\end{array}$ & $\begin{array}{l}8 \\
4 \\
5 \\
6\end{array}$ & $\begin{array}{l}2 \\
6 \\
5 \\
4\end{array}$ \\
\hline
\end{tabular}

^Number of cases.

Abbreviations: NCV, nerve conduction velocity. W-E, wrist-elbow segment. E-A, elbow-axilla segment. N, Number of tested cases.

'Normal limits for the CNAP amplitude: $10 \mu \mathrm{V}$ for median and ulnar; $5 \mu \mathrm{V}$ for sural; $1.3 \mu \mathrm{V}$ for medial plantar; $0.4 \mu \mathrm{V}$ for lateral plantar nerves. ${ }^{2}$ Normal limits for the NCV: $41.3 \mathrm{~m} / \mathrm{sec}$ for median; 39.3 for ulnar; 34.7 for sural; 32.8 for medial plantar; 24.6 for lateral plantar; 49.4 for W-E segment of median; 53.4 for E-A segment of median; $47 \cdot 5$ for W-E segment of ulnar; $48 \cdot 2$ for E-A segment of ulnar nerves.

most common a bnormality in sensory nerve conduction was slow NCV. In cases 6 and 8, the near-nerve needle test of the plantar nerve confirmed demyelinating neuropathy by showing marked slowing of sensory NCV. Mixed nerve conduction was abnormal in eight cases.

Needle EMG findings The needle EMG study was performed in distal muscles in 10 cases and in paraspinal muscles in two cases. Fibrillation and positive sharp waves were detected in four cases and fasciculation in five. Long-duration ( $>17 \mathrm{~ms}$ ) and high-amplitude $(>5 \mathrm{mV}$ ) motor unit potentials (MUP) were observed in three and eight cases respectively. Interference pattern was reduced in 10 cases. Thus the majority of cases showed evidence of chronic denervation.

Sural nerve biopsy findings Sural nerve biopsy was performed in eight cases. In case 2, only paraffin sections were available. In five cases, there was a definite decrease in the population of myelinated fibres. In no case were there any inflammatory cells or amyloid deposits. Onionbulb formation was not observed in any case. Loss of myelin was the most prominent finding in the longitudinal cuts of nerve on the frozen sections. This could be due either to demyelination or loss of large fibres. In two cases a few "myelin digestion chambers" indicating mild axonal degeneration were observed. In three cases with semithin EM sections, there was evidence of demyelination: "remyelinated fibres" in three and demyelination in one. Teased nerve fibre preparation showed segmental demyelination in $18-33 \%$ of teased nerve fibres in four cases and axonal degeneration in $0-4 \%$ in two cases. Thus, the nerve biopsy confirmed that this neuropathy was predominantly demyelinating.

Treatment In five cases, there was no evidence of recent progression at the time of initial evaluation. In three of these five cases (cases 2, 3,7 ), pain had to be controlled with strong analgesics and narcotics. In five cases in which neuropathy was progressing at the time of initial evaluation, corticosteroids were tried. In three cases, there was objective improvement (by at least 2 "disability scores") with steroids alone. In cases 8 and 10, high-dose prednisone was not effective in reversing progression, and thus azathioprine and plasmapheresis were needed to produce objective improvement. In cases 6 and 8, complete recovery occurred after one course of immunotherapy. In case 5, there were two episodes of exacerbation of neuropathy when corticosteroid dosage was reduced. In case 1 , an improved status, though not normal, was maintained even after corticosteroid was withdrawn.

\section{Discussion}

We have described ten patients with idiopathic sensory neuropathy characterised by subacute or chronic progression of neuropathy and electrophysiologic and histologic evidences of demyelination. Thus this neuropathy is termed descriptively as "chronic sensory demyelinating neuropathy" (CSDN). In view of the universal involvement of motor fibres in the nerve conduction, one could argue that this neuropathy is not purely sensory. However, following the traditional method of classification of neuropathy on the basis of clinical findings, this neuropathy is justifiably classified as sensory neuropathy.

The clinical features typical of CSDN are summarised as follows: The neurological symptoms and signs were those of a diffuse sensory neuropathy with chronic progression over months and years. The majority of patients had polyneuropathy. Pain sensation and proprioception were equally affected in majority of cases. There was no motor weakness whatsoever. Reflexes were either normal or diminished. Trigeminal involvement was rare. There was no antecedent history of infection or event. There was no family history of a similar disease. The spinal fluid protein was high in most of cases. There were no cells in any cases. Oliogoclonal band was present in half of cases.

Diffuse nerve conduction abnormalities were invariable findings. Motor as well as sensory and mixed nerve conductions were affected. Electrophysiological evidences of demyelination was observed at least in two nerves in all cases. We found that motor nerve conduction abnormalities were usually the first objective clues suggestive of demyelinating neuropathy, and thus motor nerve conduction study was the most important diagnostic test for CSDN. Neuropathy was monophasic. In many, neuropathy seemed to be stabilised after certain period of progression, spontaneously or with steroid treatment. In one case (5), there was a relapse of neuropathy with reduction of steroid dose. Thus this case became steroiddependent. Immunotherapy was effective in progressive phase of disease. Immunotherapies were effective in improving neuropathy in majority of cases during the progressive phase. Thus we believe immunotherapies are indicated in CSDN when neuropathy is progressing. Segmental demyelination is the predominant finding in the sural nerve biopsy. This finding confirmed the basic nature of this entity as demyelinating neuropathy and was the basis of the electrophysiological evidence of demyelination. 
On the basis of typical features as described above, CSDN is a distinct entity. The aetiology of CSDN is not obvious from this study. However, there is evidence to suggest an autoimmune disorder: oligoclonal bands in the spinal fluid in half of cases, responses to immunotherapy, and similarities with CIDP, a well known autoimmune disease.

Whether CSDN represents a distinct clinical entity or CIDP presenting as pure sensory neuropathy cannot be determined until the diagnostic laboratory marker is identified. We believe that CSDN represents CIDP presenting as pure sensory neuropathy. CSDN has many similarities with the classical CIDP. In both there are subacute or chronic progression of neuropathy, high spinal fluid protein, and favourable response to immunotherapy. ${ }^{5-11}$ Histopathological and electrophysiological findings in CSDN are indistinguishable from CIDP. $^{5-7912}$ In two series, sensory neuropathy was recognised in passing as a variant of CIDP and reported to constitute $5-6 \%$ of cases. ${ }^{67}$ However, no detailed description of this variant was presented. Most publications do not mention this presentation. ${ }^{812}$ In formulating the diagnostic criteria for CIDP, one group excluded sensory neuropathy from the spectrum of CIDP ${ }^{12}$ while the other group included it as a spectrum of CIDP. ${ }^{13}$ In our series, CSDN represents about $8 \%$ of the cases of CIDP.

From our study, we conclude that CSDN, a distinctly recognisable entity, is CIDP present- ing as pure sensory neuropathy. An important observation in our study is that immunotherapies are effective in the progressive phase of CSDN.

1 Joy JL, Oh SJ. Sensory neuropathy as a variant of chronic inflammatory demyelinating neuropathy. Neurology 1988;38(Suppl 1):189.

2 Oh SJ, Joy JL, Sunwoo I, Kuruoglu R. A case of chronic sensory demyelinating neuropathy responding to immunotherapies. Muscle Nerve 1992;15:255-6.

3 Oh SJ. Clinical electromyography: nerve conduction studies. Baltimore: University Park Press, 1984.

4 Oh SJ, Kim HS, Ahmad BK. Electrophysiological diagnosis of the interdigital nerves of the foot. Muscle Nerve of the interdigital
$1984 ; 7: 218-25$.

5 Oh SJ. Subacute demyelinating polyneuropathy responding to corticosteroid treatment. Arch Neurol 1978;

6 Dyck PJ, Lais AC, Ohta M, Bastron JA, Okazaki H, Groover RV. Chronic inflammatory polyradiculoneuropathy. Mayo Clin Proc 1975;50:621-37.

7 McCombe PA, Pollard JD, McLeod JG. Chronic inflammatory demyelinating polyradiculoneuropathy: a clinical and electrophysiological study of 92 cases. Brain 1987;110: 1617-30.

8 Dalakas MC, Engel WK. Chronic relapsing (dysimmune) polyneuropathy: pathogenesis and treatment. Ann Neuro 1981;9(suppl): $134-45$.

9 Prineas JW, McLeod JG. Chronic relapsing polyneuritis. $f$ Neurol Sci 1976;27:427-58.

10 Dyck JW, O'Brien PC, Oviatt KF, Dinapoli RP, Duabe JR, Bartleson JD, Mokri B, Swift T, Low PA, Windeband AJ. Prednisone improves chronic inflammatory demyelinating polyradioculo-neuropathy more than no treatment. Ann Neurol 1982;11:136-41.

11 Pollard JD. A critical review of therapies in acute and chronic inflammatory demyelinating polyneuropathy. Muscle Nerve 1987;10:214-21.

12 Barohn RJ, Kissel JT, Warmolts JR, Mendell JR. Chronic inflammatory demyelinating polyradiculoneuropathy: clinical characteristics, course, and recommendations for diagnostic criteria.

13 An Ad Hoc Subcommittee of the American Academy of Neurology AIDS Task Force. Research criteria for diagnosis of chronic inflammatory demyelinating polyneu opathy (CIDP). Neurology 1991;41:617-18. 